\title{
Compton-Like Polariton Scattering in Hyperbolic Metamaterials
}

\author{
Ivan V. Iorsh, ${ }^{1}$ Alexander N. Poddubny, ${ }^{2}$ Pavel Ginzburg, ${ }^{1,4}$ Pavel A. Belov, ${ }^{1}$ and Yuri S. Kivshar ${ }^{1,3}$ \\ ${ }^{1}$ ITMO University, St. Petersburg 197101, Russia \\ ${ }^{2}$ Ioffe Physical-Technical Institute, Russian Academy of Science, St. Petersburg 194021, Russia \\ ${ }^{3}$ Nonlinear Physics Center, Australian National University, Canberra ACT 0200, Australia \\ ${ }^{4}$ Department of Physical Electronics, Fleischman Faculty of Engineering, Tel Aviv University, Tel Aviv 69978, Israel
}

(Received 17 October 2014; published 4 May 2015)

\begin{abstract}
We study the scattering of polaritons by free electrons in hyperbolic photonic media and demonstrate that the unconventional dispersion and high local density of states of electromagnetic modes in composite media with hyperbolic dispersion can lead to a giant Compton-like shift and dramatic enhancement of the scattering cross section. We develop a universal approach to study multiphoton processes in nanostructured media and derive the intensity spectrum of the scattered radiation for realistic metamaterial structures.
\end{abstract}

Scattering is one of the most fundamental processes enabling us to probe an internal structure of matter. One of the first scattering experiments was performed by Lord Rutherford with the discovery of a structure of atoms extracted from the $\alpha$-particle scattering from a gold target [1]. More recently, scattering experiments have become the most frequently used tool in atomic and condensed matter physics [2] and related cross-disciplinary areas.

One of the main parameters that defines the probability of any scattering process is the density of available states which a scattered particle could occupy. While under frequently used conditions this physical characteristic is predefined by a scattering object, the surrounding environment could contribute significantly to the scattering process. This idea is employed frequently for manipulating the spontaneous emission of light by placing an emitter inside a cavity [3].

These concepts could be pushed further by employing metamaterials with broadband nonresonant engineering of the local density of states (LDOS), e.g., by using the properties of hyperbolic metamaterials. Hyperbolic metamaterials are a special class of composite media [4] with electromagnetic properties described by the diagonal permittivity tensor with the principal components being of the opposite signs which results in a hyperbolic shape of the isofrequency contours [5]. Practical realizations of hyperbolic media are based on metal-dielectric layered structures [6], arrays of vertically aligned metal nanorods [7], or semiconductor heterostructures [8]. One of the most intriguing properties of hyperbolic media is the nonresonant broadband enhancement of the LDOS, that is limited only by the smallest yet finite scale of the system [9] and can affect the emitters placed inside or near hyperbolic media.

While the spontaneous emission and photon absorption are the first-order perturbation processes being directly influenced by the LDOS, higher-order interactions could have a nontrivial dependence on the LDOS and, as a result, they can be tailored strongly by an electromagnetic environment, as shown both theoretically [10-12] and experimentally $[13,14]$. In this Letter, we study higherorder light-matter interaction processes and, in particular, analyze the analogue of the Compton scattering [15] for polaritons modified significantly in hyperbolic media. First, we notice that the commonly used theoretical quantum methods [16] result in unphysical divergences of both frequency shifts and scattering cross sections being inapplicable to metamaterials. Being motivated to remove these singularities, we develop a quantum formalism based on the Langevin approach. We notice that due to the inherent dispersion and losses in hyperbolic metamaterials, the scattering occurs between dressed photons, polaritons, and free carriers and this process differs substantially from the well-known Compton effect. While the account for the losses and finite period of the structure destroys the singularity appearing in the description of lossless and uniform hyperbolic media, such characteristics as frequency shift and scattering cross-section are still much larger than in the case of conventional materials.

We consider a structure shown schematically in Fig. 1(a) where a monochromatic photon is normally incident upon the a semi-infinite hyperbolic medium. The presence of an interface enables us to approach a probable experimental layout as well as highlights the impact of the geometric arrangement.

Within our theoretical approach based on the Born approximation, electrons in the conducting wires cannot play the role of scattering centers since their interaction with the electromagnetic field is accounted for by an effective negative dielectric permittivity of the wires. Thus, we should generate an additional population of free electrons in the system, e.g., by using a direct injection with an $e$ gun [17]. An alternative way is to use a heavily doped semiconductor as a matrix for the wire array [18]. Free carriers in the semiconductor matrix can play the role of 


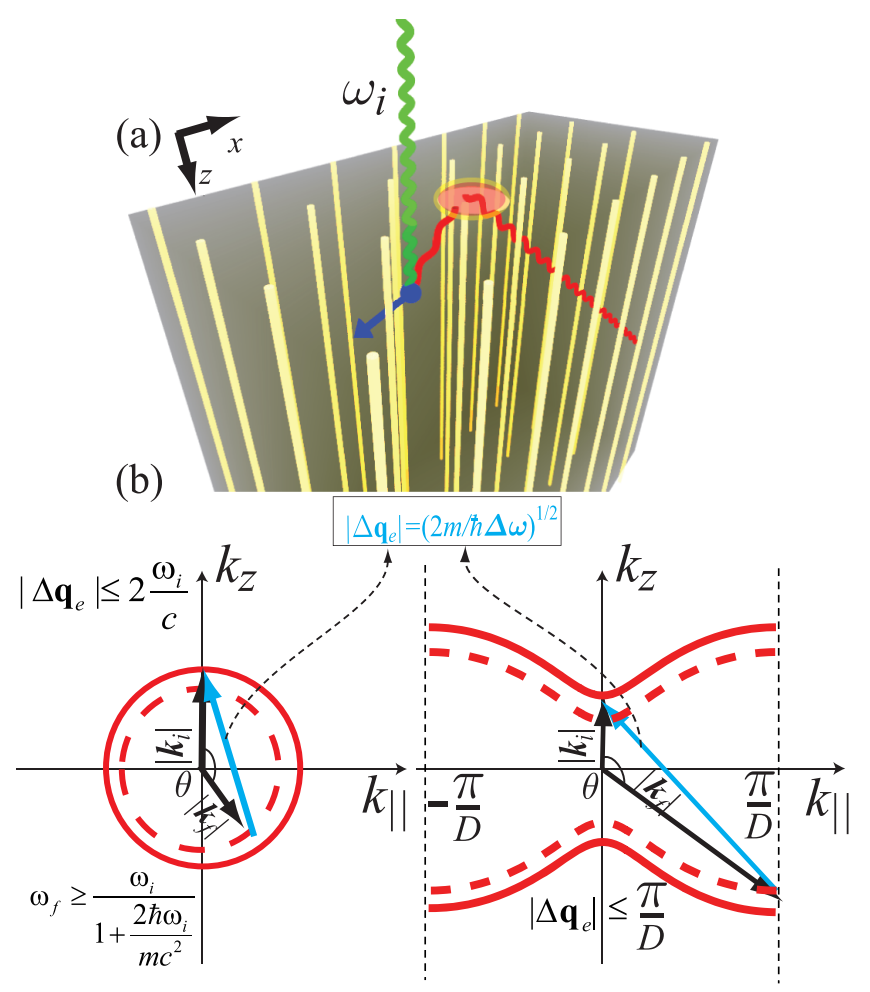

FIG. 1 (color online). (a) Schematic of the Compton-like scattering in a hyperbolic medium. An incident photon (green) is scattered (red) by a free electron (blue). (b) Diagrams illustrate phase-matching conditions, to be satisfied in vacuum (left) and a hyperbolic medium (right). Red curves correspond to the initial (solid) and scattered (dashed) photon isofrequency surfaces, blue vector shows the momentum of the electron after the scattering.

scattering centers as was shown in a number of studies [19] where the scattering of light from free carriers in semiconductors was experimentally observed.

The essence of the Compton-like polariton scattering effect can be illustrated by means of the scattering diagram shown in Fig. 1(b). If the initial momentum of an electron is zero, the momentum conservation implies that the acquired electron momentum $\Delta \mathbf{q}_{e}$ is equal to a difference between initial and final photon wave vectors. In vacuum, the wave vector of a photon is much smaller than the wave vector of an electron of the same energy and, as can be seen in Fig. 1(b), the maximum electron wave vector $\left|\Delta \mathbf{q}_{e}\right|$ is limited by $2 \omega_{i} / c$. This defines the upper bound for the electron kinetic energy, that is equal to the photon energy shift due to the energy conservation. In sharp contrast to vacuum, in a hyperbolic medium the final photon momentum is bounded only by the inverse period of the structure, leading to the large electron kinetic energy and thus the large Compton frequency shift. The scattered quanta then occupy one of the large wave vector states, the TM electromagnetic mode. Scattering to the TE eigenmodes is also allowed; however, the cross section of this process is much smaller due to the small density of the TE modes, and the frequency shifts will not be enhanced. In what follows, we consider the scattering of the TM modes only. It is worth noting that TM eigenmodes, having a $k$ vector much larger than those in the free space, will experience the total internal reflection and, as a result, will be trapped inside the hyperbolic medium. However, its near field [shown schematically with a glowing disc in Fig. 1(a)] can be detected in close proximity to the interface or inside the hyperbolic metamaterial. A proper theoretical description of the above scenario is developed below.

The permittivity tensor of the metamaterial is taken in the form $\hat{\varepsilon}=\operatorname{diag}\left(\varepsilon_{x x}, \varepsilon_{x x}, \varepsilon_{z z}\right)$, with the dispersion relation for the TM modes given by $(\omega / c)^{2}=k_{x}^{2} / \varepsilon_{z z}+k_{z}^{2} / \varepsilon_{x x}$. In order to calculate the frequency shift and differential cross section of the scattering process, we introduce the Hamiltonian for the electron system coupled to the electromagnetic field [20,21]:

$$
\begin{gathered}
\hat{H}=\hat{H}_{\mathrm{kin}}^{\mathrm{el}}+\hat{H}_{\mathrm{kin}}^{\mathrm{phot}}+\frac{e}{c} \int d \mathbf{r} \hat{\mathbf{J}} \hat{\mathbf{A}} \\
\hat{\mathbf{J}}=\frac{2 \hbar}{m} \sum_{\mathbf{k q}}\left(\mathbf{k}+\frac{\mathbf{q}}{2}\right) e^{i \mathbf{q} \mathbf{r}} \hat{c}_{\mathbf{k}}^{\dagger} \hat{c}_{\mathbf{k}+\mathbf{q}}+\frac{e}{m c} \hat{\mathbf{A}} \sum_{\mathbf{k q}} e^{i \mathbf{q} \mathbf{r}} \hat{c}_{\mathbf{k}}^{\dagger} \hat{c}_{\mathbf{k}+\mathbf{q}}
\end{gathered}
$$

where $\hat{H}_{\text {kin }}^{\text {el }}$ and $\hat{H}_{\text {kin }}^{\text {phot }}$ are electron kinetic energy operator and free electromagnetic field energy operator, respectively; where $\hat{c}_{k}, \hat{c}_{k}^{\dagger}$ are the annihilation and creation operators for the electron with the momentum $k$, and $\hat{\mathbf{A}}$ is the vector potential operator of the electromagnetic field in the second quantized form. The first term in Eq. (2) is the paramagnetic current which corresponds to the process of absorption (or emission) of the photon by an electron, which is forbidden for free electrons in special relativity. Thus, we account for the second, diamagnetic term in Eq. (2) only. Scattering of a single photon by a single electron is a textbook problem [16], and the scattering cross section is given by

$$
\begin{aligned}
\sigma_{\mathrm{TM}}= & r_{c}^{2} \iint k_{f}^{2} d k_{f} d \Omega \frac{\left|\mathbf{e}_{k_{i}} \mathbf{e}_{k_{f}}\right|^{2} c^{2}}{\omega_{i} \omega_{f}} \\
& \times \delta\left(\frac{\omega_{\mathrm{el}}+\Delta \omega}{c}-\sqrt{\left(\frac{\omega_{\mathrm{el}}}{c}\right)^{2}+\Delta k^{2}}\right),
\end{aligned}
$$

where $\quad \omega_{\mathrm{el}}=m c^{2} / \hbar, \quad \Delta \omega=\omega_{i}-\omega_{f}, \quad \Delta k=$ $\sqrt{k_{i}^{2}+k_{f}^{2}-2 k_{i} k_{f} \cos \theta}, k_{i}=\sqrt{\varepsilon_{x x}} \omega_{i} / c$ and $k_{f}$ are initial and final photon momenta, $\mathbf{e}_{k_{i}, f}$ are the polarization vectors for the initial and scattered photon, $d \Omega=\sin \theta d \theta d \phi$ is the differential of the solid angle, $r_{c}=e^{2} /\left(m c^{2}\right)$ is the classical electron radius, and the delta function in the expression Eq. (3) ensures the energy conservation in the system, and it defines the scattered photon frequency $\omega_{f}$. We assume that the electron was initially at rest. 

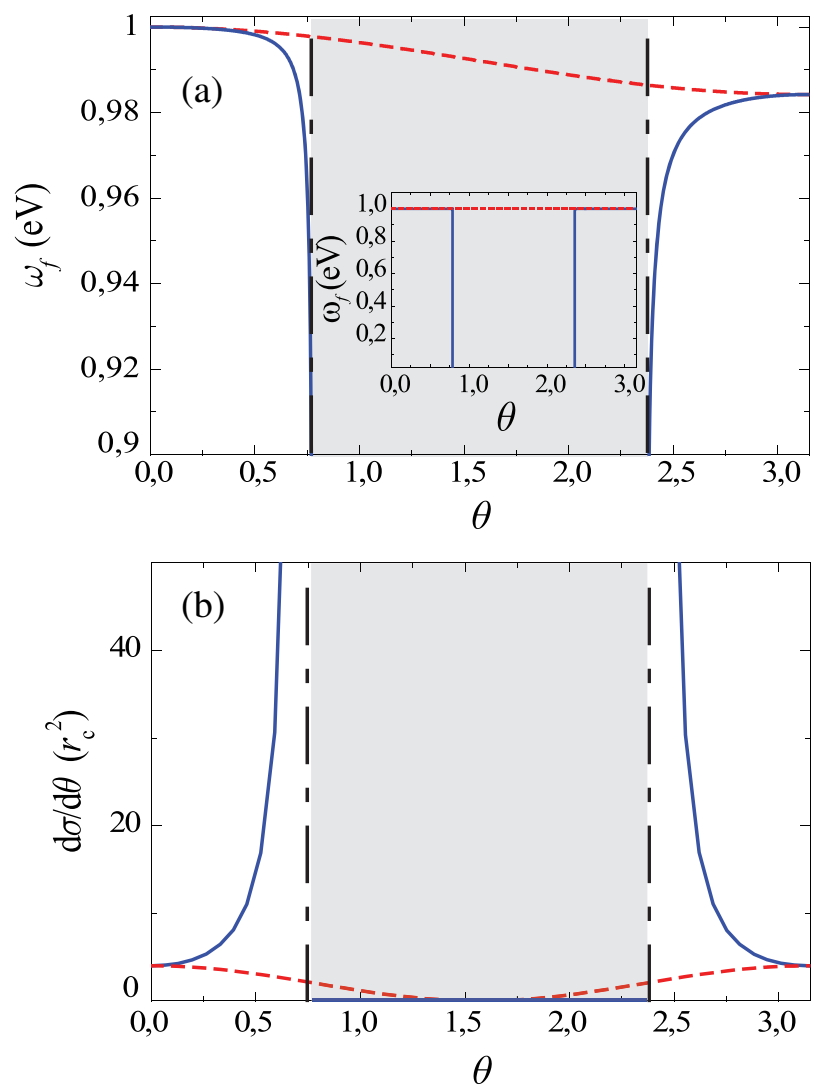

FIG. 2 (color online). (a) Frequency of the photon and (b) differential cross section for the Compton-like scattering in a lossless dielectric medium with permittivity $\varepsilon=4$ (red dashed curves) and in a hyperbolic medium with permittivity $\varepsilon_{x x}=4, \varepsilon_{z z}=-4$ (blue solid curves). The initial photon frequency is $1 \mathrm{eV}$.

The plots of the scattered photon frequency and differential cross section in an ideal case of a uniform lossless hyperbolic medium are presented in Figs. 2(a) and 2(b). We notice that as the scattering angle approaches the value $\theta_{\mathrm{cr}}=\cot ^{-1}\left(\sqrt{\varepsilon_{x x} / \mid \varepsilon_{z z}} \mid\right)$ the scattered photon frequency vanishes, and the differential cross section diverges. The scattering cross section vanishes exactly for the scattering angles $\theta_{\mathrm{cr}}<\theta<\pi-\theta_{\mathrm{cr}}$. This can be easily explained if we recall that for these angles Green's function is evanescent in lossless hyperbolic media [22]. As for the case of the Purcell enhancement, the divergencies can be overcome if we account for a finite period of the structure. Simple calculations suggest that the Compton shift enhancement is proportional to $(\lambda / D)^{2}$, where $\lambda$ is the wavelength and $D$ is the period of the structure, and the cross-section enhancement, just as the density of states, is proportional to $(\lambda / D)^{3}$.

To evaluate the experimental feasibility of the suggested effect, we consider a more realistic case of a lossy and bounded metamaterial. We employ the Langevin approach when the problem is fully characterized by Green's function determining the electromagnetic response to local currents [20]. The calculation procedure involves three steps: (i) calculation of a wave transmitted inside the medium; (ii) determination of the local currents arising due to the wave scattering by electrons, and (iii) reconstruction of the scattered wave from the known currents. A similar approach has been recently applied to the Brillouin scattering of exciton-polaritons in semiconductor superlattices [23].

First, we consider an electromagnetic plane wave incident normally upon an interface of a semi-infinite hyperbolic medium. The vector potential of the incident wave in the hyperbolic medium can be written as $\mathbf{A}(z, t)=$ $\mathbf{e}_{0} A_{0} t_{0} \exp \left[-i \omega_{i} t+i k_{0 z}^{h} z\right]$, where $\mathbf{e}_{0}$ is the polarization basis vector, $\omega_{i}$ is the initial photon frequency, $t_{0}$ is the transmission coefficient for the normal incidence, and $k_{0 z}^{h}=\sqrt{\varepsilon_{x x}} \omega_{i} / c$.

Second, in order to consider the scattering process we account for the incident field interaction with an ensemble of free electrons inside the hyperbolic media. The incident wave excites a current described by the following density operator: $\hat{\mathbf{j}}(\mathbf{r}, t)=e^{2} /$ $\left(m c^{2} V\right) \sum_{\mathbf{q}_{i}, \mathbf{q}_{f}} \hat{c}_{\mathbf{q}_{f}}^{\dagger} \hat{c}_{\mathbf{q}_{i}} e^{i\left(\mathbf{q}_{i}-\mathbf{q}_{f}\right) \mathbf{r}-i(\hbar / 2 m)\left(q_{i}^{2}-q_{f}^{2}\right) t} \mathbf{A}(z, t) \quad$ [21], that corresponds to the second, diamagnetic, term in Eq. (2). Here $V$ is the normalization volume. The (scattered) electric field operator at the position $z_{0}$ reads

$$
\hat{\mathbf{E}}(\boldsymbol{r}, t)=\frac{1}{c^{2}} \int \overline{\bar{G}}\left(\boldsymbol{r}, \mathbf{r}^{\prime}, t-t^{\prime}\right) \frac{\partial \hat{\mathbf{j}}\left(\mathbf{r}^{\prime}, t^{\prime}\right)}{\partial t^{\prime}} d^{3} \mathbf{r}^{\prime} d t^{\prime}
$$

where $\overline{\bar{G}}$ is a dyadic Green's function for the semi-infinite hyperbolic medium. In the case when the source is placed inside a hyperbolic medium and the observation point is outside, at the distance $z_{0}$ from the interface, the Green's tensor reads [24]

$$
\begin{aligned}
\overline{\bar{G}}\left(\boldsymbol{\rho}, z_{0}, \boldsymbol{\rho}^{\prime}, z^{\prime}, \omega\right)= & \int \frac{i d^{2} \mathbf{k}_{\rho}}{(2 \pi)^{3} k_{z}} e^{i \mathbf{k}_{\rho}\left(\boldsymbol{\rho}-\boldsymbol{\rho}^{\prime}\right)-i\left(k_{z} z^{\prime}+k_{z}^{V} z_{0}\right)} \\
& \times \sum_{\lambda=\mathrm{TE}, \mathrm{TM}} t_{h V}^{\lambda} \mathbf{e}_{h, \lambda} \otimes \mathbf{e}_{V, \lambda},
\end{aligned}
$$

where $k_{z}=\left[\varepsilon_{x x}(\omega / c)^{2}-\left(\varepsilon_{x x} / \varepsilon_{z z}\right) k_{\rho}^{2}\right]^{1 / 2}, k_{z}^{V}=\left[(\omega / c)^{2}-\right.$ $\left.k_{\rho}^{2}\right]^{1 / 2}$ is the normal component of the wave vector in vacuum, $k_{\rho}$ is the in-plane photon wave vector, $t_{h V}^{\sigma}$ is the transmission coefficient, and $\mathbf{e}_{h}, \mathbf{e}_{V}$ are the polarization basis vectors in the hyperbolic media and vacuum, respectively.

Finally, by performing the spatial and time Fourier transformations, we obtain the intensity of the scattered polaritons averaged over the electron distribution,

$I\left(\mathbf{k}_{\rho}, z_{0}, \omega\right)=\int d t e^{i \omega t}\left\langle\hat{\mathbf{E}}\left(\mathbf{k}_{\rho}, z_{0}, t\right) \cdot \hat{\mathbf{E}}^{\dagger}\left(\mathbf{k}_{\rho}, z_{0}, 0\right)\right\rangle$.

The resulting correlators have the form $\mathcal{P}=$ $\left\langle\hat{c}_{\mathbf{q}_{f}}^{\dagger} \hat{c}_{\mathbf{q}_{i}} \hat{c}_{\mathbf{q}_{\mathbf{i}^{\prime}}}^{\dagger} \hat{c}_{\mathbf{q}_{f^{\prime}}}\right\rangle$ and can be straightforwardly evaluated by using Wick's theorem: $\mathcal{P}=\delta_{f f^{\prime}} \delta_{i i^{\prime}} n_{i}\left(1-n_{f}\right)+$ $\delta_{i f} \delta_{i^{\prime} f^{\prime}} n_{i} n_{i^{\prime}}$. 
The second term corresponds to the Rayleigh scattering with the conservation of frequency and in-plane wave vector. Here we focus on the first term, that is responsible for the inelastic scattering. The expression for the spectral density function can be written as

$$
I\left(\omega, k_{\rho}\right)=E_{0}^{2}\left(\frac{\omega}{\omega_{i}}\right)^{2} \frac{16 \pi t_{0}^{2} r_{c}^{2} V^{2 / 3} m}{\varepsilon_{x x} \hbar} \mathcal{I}\left(\omega, k_{\rho}\right),
$$

where the dimensionless factor $\mathcal{I}$ is given by the expression

$$
\begin{aligned}
\mathcal{I}\left(\omega, k_{\rho}\right)= & \frac{e^{-2 \operatorname{Im} k_{z}^{V} z_{0}}}{\left|1+\frac{k_{z}}{k_{z v} \varepsilon_{x x}}\right|^{2}} \int \frac{d^{3} q_{i} n_{i}\left(1-n_{f(i)}\right)}{\left|\tilde{q}_{f z}\right|} \\
& \times \sum_{\xi= \pm 1} \frac{1}{\left[q_{i z}+\left(k_{0 z}-\operatorname{Re} k_{z}\right)+\xi \tilde{q}_{f z}\right]^{2}+\operatorname{Im} k_{z}^{2}},
\end{aligned}
$$

and $\quad \tilde{q}_{f z}=\left[q_{i z}^{2}+\frac{2 m}{\hbar}\left(\omega-\omega_{i}\right)-k_{\rho}^{2}+2 q_{i \rho} k_{\rho} \cos (\delta \phi)\right]^{1 / 2}$, where $q_{i \rho}$ is the in-plane component of the electron before scattering, $\delta \phi$ is the angle between the in-plane wave vector of the incident radiation and in-plane wave vector of the electron, $n_{i, f}$ are the Fermi distribution functions, and $n_{f(i)}$ means that the electron final energy is determined by the conservation laws $E_{f}^{\mathrm{el}}=\left(\hbar^{2} / 2 m\right)\left[\tilde{q}_{f z}^{2}+\left(\mathbf{q}_{i \rho}-\mathbf{k}_{\rho}\right)^{2}\right]$. The dimension of $I$ in Eq. (8) is volume energy density per frequency per two-dimensional wave vector, and thus it corresponds to the intensity of the reflected field at the fixed frequency and wave vector. This value is directly proportional to the differential cross section per unit area of a metamaterial surface.

The integral in Eq. (7) can be calculated analytically in the special case of zero temperature and small carrier concentration $n_{\mathrm{el}}$, when the Fermi wave vector $k_{F}=\left(3 \pi^{2} n_{\mathrm{el}}\right)^{1 / 3}$ is much less than $\Delta=\sqrt{2 m\left(\omega_{i}-\omega\right) / \hbar}$. For the Compton shifts $\hbar\left(\omega_{i}-\omega\right)$, as small as $0.01 \mathrm{meV}$, this regime is realized for the electron densities up to $n_{\mathrm{el}} \approx 4 \times 10^{15} \mathrm{~cm}^{-1}$ and $k_{F} \approx 4.9 \times 10^{5} \mathrm{~cm}^{-1}$. Below, we demonstrate that the actual values of the Compton shift can be much larger, so the low-density approximation may work even better. The dimensionless integral in Eq. (8) is then given by

$\frac{1}{\mid \sqrt{\Delta^{2}-k_{\rho}^{2}}} \sum_{\xi= \pm 1} \frac{4 \pi k_{F}^{3} / 3}{\left(k_{0 z}-k_{z z}+\xi \sqrt{\Delta^{2}-k_{\rho}^{2}}\right)^{2}+\operatorname{Im} k_{z z}^{2}}$.

In Fig. 3 we plot the dimensionless part of the scattered intensity [Eq. (8)] for a hyperbolic medium with $\varepsilon_{x x}=4.0$, $\varepsilon_{z z}=-4.0+0.3 i$ [see Fig. 3(a)], and a dielectric medium with the permittivity $4.0+0.3 i$ [see Fig. 3(b)]. Figure 3(c) shows the integrated spectral intensity as a function of the Compton shift for both dielectric and hyperbolic media. The dielectric permittivity dispersion could be neglected, since we are working in a narrow frequency range. The intensity of the scattered radiation is calculated at the distance of $20 \mathrm{~nm}$ from the surface of the structure.
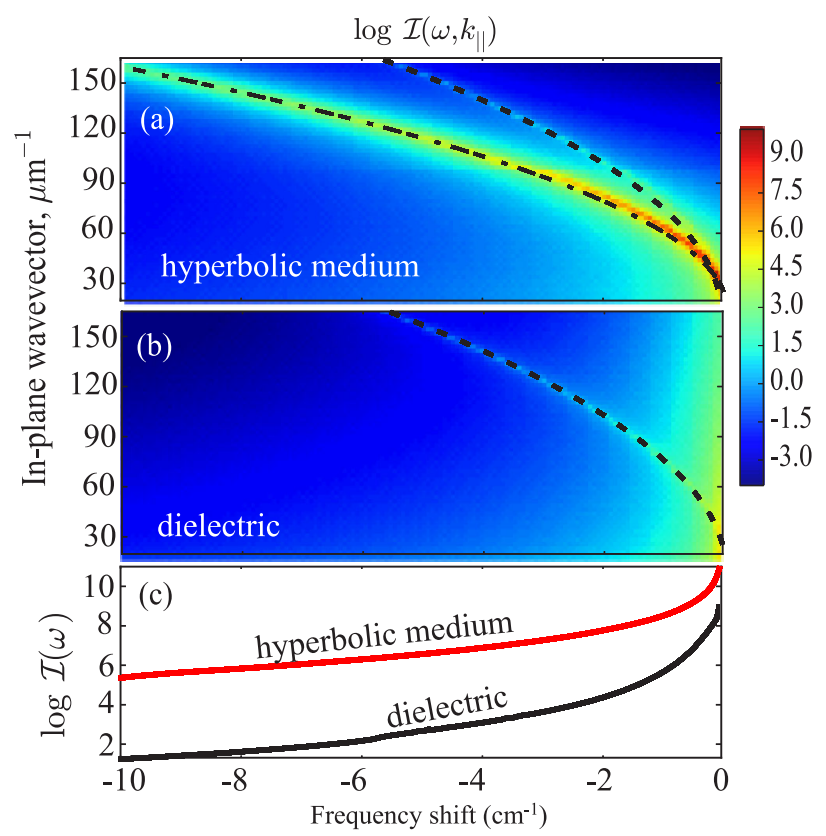

FIG. 3 (color online). (a),(b) Logarithmic map of the dimensionless part of the spectral density $I$ vs in-plane wave vector and the frequency shift for the case of (a) hyperbolic and (b) isotropic dielectric media. (c) Integral of the spectral density over the inplane momenta.

The Fermi wave vector is equal to $10^{5} \mathrm{~cm}^{-1}$. For the case of light scattering by an electron gas rather than an isolated free electron, the value of the shift increases with the Fermi wave vector of the gas: $\delta \omega \sim \hbar^{2} / m k_{0}\left(k_{0} k_{F}+k_{0} / 2\right), k_{0}=$ $\omega_{i} / c$ [19]. To isolate the contribution of the modified photonic density of states from the overall shift enhancement, we assume the same electron distributions for the case of dielectric and hyperbolic media which correspond experimentally to the case of a bulk semiconductor and a metallic wire array grown in the same semiconductor matrix [18].

Our formalism also allows us to account for a periodicity of the structure. In periodic structures, the in-plane wave vector $k_{\rho}$ cannot exceed $2 \pi / D$. Thus, in Figs. 3(a) and 3(b) we extend the axis $k_{\rho}$ only to $150 \mu \mathrm{m}^{-1}$ which corresponds to the period of $20 \mathrm{~nm}$.

The spectra of the scattered radiation shown in Figs. 3(a) and 3(b) and calculated numerically with Eq. (8), are well approximated by the analytical result of Eq. (9). Singularities in Eq. (9) describe different scattering resonances. Two scattering channels can be distinguished in Figs. 3(a) and 3(b). The first channel is marked by a dashed curve, and it corresponds to a pole of the first factor of Eq. (9). The Compton shift is defined as $\omega_{i}-\omega_{f}=$ $\hbar k_{\rho}^{2} /(2 m)$, i.e., the scattered electron propagates along an interface with vacuum. This interface feature is similar to the van Hove singularity in the electronic density of states of one-dimensional systems [25], or Wood's anomalies in the grating diffraction [26]. While it extends up to the large wave vectors, it is integrable and does not contribute much to the overall scattered intensity. 
For hyperbolic media, another scattering channel becomes dominant, and it is marked by a dash-dotted curve in Fig. 3(b). This channel corresponds to the resonance in the second factor of Eq. (9) described by the bulk momentum conservation law. Large in-plane wave vectors of scattered photons and electrons mean larger scattered electron kinetic energies and, as a result, larger Compton shifts. For dielectric media, this scattering channel does not exist because the photon modes with large in-plane wave vectors are not available, see Fig. 1(b). Figure 3(c) demonstrates the main result of our analysis: the scattering becomes much stronger in the hyperbolic regime so that the Compton shifts up to $\sim 10 \mathrm{~cm}^{-1}$, which is of the order of $1 \mathrm{meV}$ can be attained.

The use of hyperbolic media could facilitate a detection of very weak nonlinear processes, similar to the case of rough plasmonic surfaces enabling the detection of the Raman scattering via a local field enhancement [27]. Giant Compton-like shifts and enhanced scattering cross sections could be detected via near-field imaging, either inside a bulk structure or close to a surface. Also, the internal structure of hyperbolic media leads to local field corrections, since the value of the Compton shift and cross section depend on the exact position of electron. However, if the spatial distribution of the free electrons is quasiuniform, then the local field corrections can be averaged and the effective medium approximation can be applied [28]. A similar effect appears in extremely anisotropic metamaterials with positive dielectric permittivities which can be realized with plasmonic periodic structures [29].

In conclusion, we have developed a general formalism for describing inelastic scattering processes in hyperbolic metamaterials. In particular, we have demonstrated theoretically that the Compton-like polariton scattering in hyperbolic media differs substantially from the well-known Compton scattering in vacuum, and we have predicted that Compton shifts for the visible light can be enhanced to become observable in realistic experiments. The concept of inelastic scattering enhancement in hyperbolic media is rather general, and it can be extended to other types of quasiparticles excited in media with hyperbolic dispersion, including phonons and surface plasmons.

The work was supported by the Ministry of Education and Science of the Russian Federation (Zadanie No. 3.1231.2014/K), the Russian Foundation for Basic Research, and the Australian Research Council through the ARC Center of Excellence CUDOS. Grants of the President of Russian Federation, MK-5220.2015.2, MD7841.2015.2, and Dynasty foundation.

[1] E. Rutherford, The Scattering of Alpha and Beta Particles by Matter and the Structure of the Atom (Taylor \& Francis, London, 2011).
[2] M. Cardona and M. Brodsky, Light Scattering in Solids I (Springer, New York, 2011).

[3] E. M. Purcell, Phys. Rev. 69, 674 (1946).

[4] D. R. Smith and D. Schurig, Phys. Rev. Lett. 90, 077405 (2003).

[5] A. N. Poddubny, I. I. Iorsh, P. A. Belov, and Y. Kivshar, Nat. Photonics 7, 948 (2013).

[6] A. J. Hoffman, L. Alekseyev, S. S. Howard, K. J. Franz, D. Wasserman, V. A. Podolskiy, E. E. Narimanov, D. L. Sivco, and C. Gmachl, Nat. Mater. 6, 946 (2007).

[7] A. V. Kabashin, P. Evans, S. Pastkovsky, W. Hendren, G. A. Wurtz, R. Atkinson, R. Pollard, V. A. Podolskiy, and A. V. Zayats, Nat. Mater. 8, 867 (2009).

[8] C. Rizza, A. Ciattoni, E. Spinozzi, and I. Columbo, Opt. Lett. 37, 3345 (2012).

[9] A. N. Poddubny, P. A. Belov, P. Ginzburg, A. V. Zayats, and Y. S. Kivshar, Phys. Rev. B 86, 035148 (2012).

[10] D. de Ceglia, M. A. Vincenti, S. Campione, F. Capolino, J. W. Haus, and M. Scalora, Phys. Rev. B 89, 075123 (2014).

[11] C. Argyropoulos, N. M. Estakhri, F. Monticone, and A. Alu, Opt. Express 21, 15037 (2013).

[12] A. N. Poddubny, P. Ginzburg, P. A. Belov, A. V. Zayats, and Y. S. Kivshar, Phys. Rev. A 86, 033826 (2012).

[13] L. Gu, T. Tumkur, G. Zhu, and M. Noginov, Sci. Rep. 4, 4969 (2014).

[14] M. Mayy, G. Zhu, A. Webb, H. Ferguson, T. Norris, V. Podolskiy, and M. Noginov, Opt. Express 22, 7773 (2014).

[15] M. Cooper, P. Mijnarends, N. Shiotani, N. Sakai, and A. Bansil, X-Ray Compton Scattering (Oxford University Press, New York, 2004).

[16] W. Greiner, Quantum Mechanics: Special Chapters (Springer-Verlag, Berlin, 1998).

[17] G. Adamo, K. F. MacDonald, N. I. Zheludev, Y. H. Fu, C-M. Wang, D. P. Tsai, and F. J. Garcia de Abajo, Phys. Rev. Lett. 103, 113901 (2009).

[18] A. Atrashchenko, A. Nashchekin, M. Mitrofanov, V. P. Ulin, and V. P. Evtikhiev, Phys. Staus Solidi-Rapid Research Lett. 8, 325 (2014).

[19] A. Mooradian, Phys. Rev. Lett. 20, 1102 (1968).

[20] C. Raabe, S. Scheel, and D.-G. Welsch, Phys. Rev. A 75, 053813 (2007).

[21] H. Bruus and K. Flensberg, Many-Body Quantum Theory in Condensed Matter Physics (Oxford University Press, New York, 2004).

[22] A. S. Potemkin, A. N. Poddubny, P. A. Belov, and Y. S. Kivshar, Phys. Rev. A 86, 023848 (2012).

[23] A. N. Poddubny, A. V. Poshakinskiy, B. Jusserand, and A. Lemaitre, Phys. Rev B 89, 235313 (2014).

[24] L. Novotny and H. Brecht, Principles of Nanooptics (Cambridge University Press, Cambridge, England, 2006).

[25] C. Kittel, Introduction to Solid State Physics (Wiley, New York, 2006).

[26] A. Hessel and A. A. Oliner, Appl. Opt. 4, 1275 (1965).

[27] M. Kauranen and A. V. Zayats, Nat. Photonics 6, 737 (2012).

[28] A. N. Poddubny, P. A. Belov, and Y. S. Kivshar, Phys. Rev. B 87, 035136 (2013).

[29] A. Orlov, S. Zhukovsky, I. Iorsh, and P. Belov, Photonics and Nanostructures: Fundamentals and Applications 12, 213 (2014). 